\title{
Remote Patient Management for Emerging Geographical Areas
}

\author{
Xueqing $Y u^{a-c} \cdot$ Xiao Yang ${ }^{a, b}$
}

a Department of Nephrology, The First Affiliated Hospital, Sun Yat-sen University, Guangzhou, China; ${ }^{b}$ Key Laboratory of Nephrology, National Health Commission and Guangdong Province, Guangzhou, China; ' Guangdong Provincial People's Hospital, Guangdong Academy of Medical Science, Guangzhou, China

\begin{abstract}
The increase in end-stage renal disease (ESRD) worldwide is of great concern for many countries and has become a worldwide public health problem, which puts a burden on medical healthcare resources. The geographic variation of ESRD has been observed both locally and globally. Greater distance care facilities may impede timely delivery of good quality care and consequently increase the risk of mortality as well as decreased health-related quality of life. Patients living in remote and rural areas could use peritoneal dialysis (PD) as a home-based and economical therapy option. However, morbidity and mortality in PD patients at remote region have been reportedly increasing. Monitoring and managing PD patients living in remote or underserved areas is a big challenge. The establishment and practice of PD satellite center model in China for the management of remote PD patients are a concern for peer professionals across the world. With the support of remote PD center programs, such as the PD satellite center model and telehealth and so on, home PD therapy have further enhanced the PD technology, patient management exercised by quality PD centers, which accordingly benefit more ESRD patients who live in remote areas. Such PD programs can mitigate patients' financial, travel and healthcare pressure and to a certain extent improve the outcomes of these PD patients. Further research is needed to explore more interventions in the management of remote PD patient and better understand the factors that affect the success of these interventions.


The prevalence of end-stage renal disease (ESRD) is increasing all over the world. According to the United States Renal Data System, the number of incident ESRD cases was 124,675 and the unadjusted incidence rate was 373.4 per million population (pmp) in 2016. The number of prevalent ESRD cases has continued to rise by about 20,000 cases per year in USA [1]. Mainland China also confronts this severe challenge. Chronic kidney disease (CKD) is a precursor to ESRD. Results of a multi-center cross-sectional CKD study showed that the prevalence of CKD is $10.8 \%$ [2], and it was estimated that the prevalence of ESRD in China is about 200-250 cases pmp. The prevalence is projected to rise rapidly, fueled by aging population and the high prevalence of related primary chronic disease, such as diabetes and hypertension [3]. The sheer increasing incidence of ESRD has a major impact on the need for dialysis, including maintenance hemodialysis (HD), peritoneal dialysis (PD), for maintenance of life. In USA, the number of patients on HD was 107,225 and on PD was 11,965 in 2016 [1]. Renal replacement therapy in Europe had a prevalence of $801 \mathrm{pmp}$ in 2015 [4]. The overall incidence of treated ESRD in Australia has increased by $19 \%$ between 2000 and 2007 [5]. China is the biggest developing country with vast area and the biggest population ( $>1.3$ billion) in the world. Due to China's limited economic and medical resources, coupled with limited health insurance coverage, the prevalence of dialysis is very low in China compared with other developed countries [3]. According to the Chinese Renal Data System, there were 596,365 patients registered on dialysis at the end of 2017 in China [6]. ESRD is largely responsible for the cost of health systems, with an annual growth in dialysis programs ranging from 6 to $12 \%$ in the last 2 decades [7]. Although ESRD patients represent only $0.15 \%$ of the total population in Taiwan of China, they are responsible for 7\% of the total annual budget of Taiwan's NHI Program owing to their use of dialysis [8]. A similar trend is observed in USA where ESRD is responsible for $7.2 \%$ of the total annual budget of USA's overall Medicare paid claims [1]. Therefore, the increase in ESRD populations worldwide is of great concern for many countries and has become a worldwide public health problem and puts a heavy medical burden on healthcare resources.

\section{Prevalence and Outcomes of Remote ESRD Patients}

The geographic variation of ESRD has been observed both locally and globally. It was found that more remote communities accessible only by air had a higher prevalence of CKD (34.4\%) than communities accessible by road (17.6\%), a 2-fold higher prevalence of $\mathrm{CKD}$ in indigenous Canadians in comparison to the general population [9]. The number of rural indigenous patients with kidney failure re- 
quiring dialysis has increased disproportionately in Canada over the last 25 years [10]. A study identified a clear and significant regional difference among rural and urban counties in South Carolina, where the incidence rates of ESRD were consistently higher in rural than in urban counties [11]. Colombia has one of the largest percentages of rural populations among the countries in Latin America, with $31 \%$ living outside larger cities and towns [12]. In Australia, many indigenous patients reside away from city centers [5]. In a rural and urban epidemiology survey in Guangdong Province of China, we found that the prevalence of CKD in urban populations is $10.1 \%$ [13] while in rural area it is up to $13.6 \%$ [14].

Remote dwellers must travel long distances to care facilities and experience barriers to healthcare delivery such as lower income, geographical isolation, and imbalances in physician supply, which leads to lower patient-physician ratios and lower quality of care [15]. Most patients with ESRD are treated with renal replacement therapy, including $\mathrm{HD}$ or $\mathrm{PD}$ performed in medical facilities or at home. Both modalities require ongoing supervision by nephrologists to adjust the dialysis prescription, manage complications, and maximize outcomes. However, dialysis clinics are typically located in large urban areas. Most dialysis patients who lived in remote rural areas or small towns had to travel to urban renal dialysis clinics for treatment or follow-up care. Studies have explored the association between the distance from the facility and health outcomes among ESRD patients. Canadian data have shown that people with kidney disease who live more than $50 \mathrm{~km}$ from a nephrologist have poorer outcomes than those who live less than $50 \mathrm{~km}$ away [16]. Similarly, a distance of more than $50 \mathrm{~km}$ was associated with an increased risk of death for dialysis patients [17, 18]. Those remote patients are less likely to receive specialist care, recommended laboratory testing, and appropriate medications. Higher rates of mortality have also been reported in China for ESRD patients who live in rural areas compared with those living in urban areas. These findings were associated with a number of factors such as relatively poor financial status, lack of or inadequate medical insurance, poorer education as well as less availability of nephrologists and necessary dialysis facility in the rural areas [19]. The effect of rurality varied with the distance. It was reported that although patients living in remote areas were more likely to live in a rural area, half of the patients in the study who lived more than $160 \mathrm{~km}$ from the renal center resided in an urban setting, suggesting that distance rather than rural or urban status had a greater effect on the risk of mortality in that cohort of patients in the United States [20]. Greater distances to care facilities may impede the timely delivery of good quality care and consequently increase the risk of mortality as well as decreased health-related quality of life. The relationship of remoteness, rurality, and ESRD outcomes has been of immense interest in the last few decades. 


\section{PD in Remote ESRD Patients and Challenges for the Management}

Compared to in-center HD, PD has many inherent advantages, such as homebased therapy, superior residual renal function preservation, better hemodynamics maintenance, and higher quality of life. The utilized medical resources, including manpower, space, and medical equipment are relatively minimal [3]. In most countries, the cost of home therapies such as PD is less and thus, any mechanisms that can increase the uptake of home therapies are likely to result in significant cost savings [21]. Continuing improvements in clinical outcomes and demonstration of the socioeconomic benefits of PD have resulted in the rapid growth of the PD population. By latest estimates, PD has currently been used as a chronic life-sustaining treatment by more than 272,000 ESRD patients, or $11 \%$ of the global dialysis population. PD use is increasing dramatically in developing countries, with a 2.50-times increase in PD prevalence over 12 years [22].

Patients living in remote and rural locations could use PD as a home-based treatment option. Since PD patients typically perform the treatment themselves in their own homes, they tend to be more flexible than those treated with HD, who typically must travel to a healthcare facility at least two-three times weekly to receive their dialysis treatment. In Australia, there was an increase in the proportion of indigenous patients commencing PD from 14\% in 2004 to $19 \%$ in 2008. Of indigenous patients initiated on PD, a greater proportion of these patients were located within remote $(79 \%)$ compared to regional (9\%) or metropolitan (8\%) locations. Analysis of the Australia and New Zealand Dialysis and Transplant (ANZDATA) Registry demonstrated that PD patients residing in non-metropolitan locations, especially remote indigenous patients have a greater risk of $\mathrm{PD}$-associated complications including peritonitis, all-cause and peritonitis-related mortality [23]. In rural regions of the USA, an increased rate of PD has also been shown despite rural facilities being less likely to offer PD training. It was no surprise that rural units with $\mathrm{PD}$ programs have worse overall outcomes [24]. Recently, PD is developing rapidly in the mainland of China and has registered 86,264 PD patients by the end of 2017 [6]. However, this expansion is limited to large cities as there are not enough well-trained PD physicians and nurses in rural areas [3]. Canada is a large country, and Canadian nephrologists tend to be concentrated in major urban centers. According to the Canadian Organ Replacement Registry, remote-location ESRD patients were more likely to initiate PD, especially Aboriginal patients living more than $300 \mathrm{~km}$ from their nephrologist to avoid relocation to an area served by a HD unit [18]. However, remote locations in geographically diverse nations such as Canada often have fewer primary care physicians and existing providers face greater challeng- 
es in delivering comprehensive PD care. An association between remoteness and increased mortality was demonstrated among Canadian PD patients, with a $15 \%$ increased risk for death among patients living more than $300 \mathrm{~km}$ away [18]. Increased distance could lead to deficiencies in PD training, dietary education, and response time for complications. Our previous study demonstrated that more frequent face-to-face patient-doctor contact (PDC) lead to better patient outcomes in PD patients [25]. The rate of PD was higher in remote regions, reflecting the benefits of home therapies in this population. However, due to their frequency of care, patients with PD often need to contact with healthcare providers across several points of care to monitor and manage their health, which can be challenging and resource intensive, particularly when patients live in remote or underserved areas.

\section{Strategy of Managing Remote ESRD PD Patients}

For the emerging big population of remote PD patients, remote monitoring systems such as mobile phone, telemedicine and network access, and so on, have been suggested and practiced in some PD centers in recent years, which may improve some processes of care and to a certain extent improve the outcomes of remote $P D$ patients when used in addition to standard ESRD care $[21,26]$. Here we share our experiences on how to manage remote PD patients.

\section{PD Satellite Center Model for the Management of Remote PD Patients}

Although utilization of PD has increased rapidly in developing countries, this expansion appears to occur mainly in city environments. Our PD center is located in Guangzhou City, which is the capital city of the Chinese province of Guangdong. Since 1978, we have used PD technology in the treatment of ESRD and promoted it throughout the nation by means of the in-service program for advanced nephrologists organized by the Ministry of Health. We established standardized management procedures for PD, regarding the placement of PD catheter, patient training and follow-up visits, collection and analysis of clinical data. In addition, quality improvement and re-assessment continued. More than 1,000 PD patients have been followed up in our center since 2015. However, in our program, over $60 \%$ of the PD patients lived outside the city of Guangzhou where the quality of $\mathrm{PD}$ care was inadequate, that is, small scale, poor technique, and non-standard education, thus leading to a lower patient survival rate, high peritonitis rate, and high drop-out rate. These conditions were challenging for the well management of these patients. To address this imbalance, our center successfully established a PD satellite center program across Guangdong Province in 2008, with the aim to 


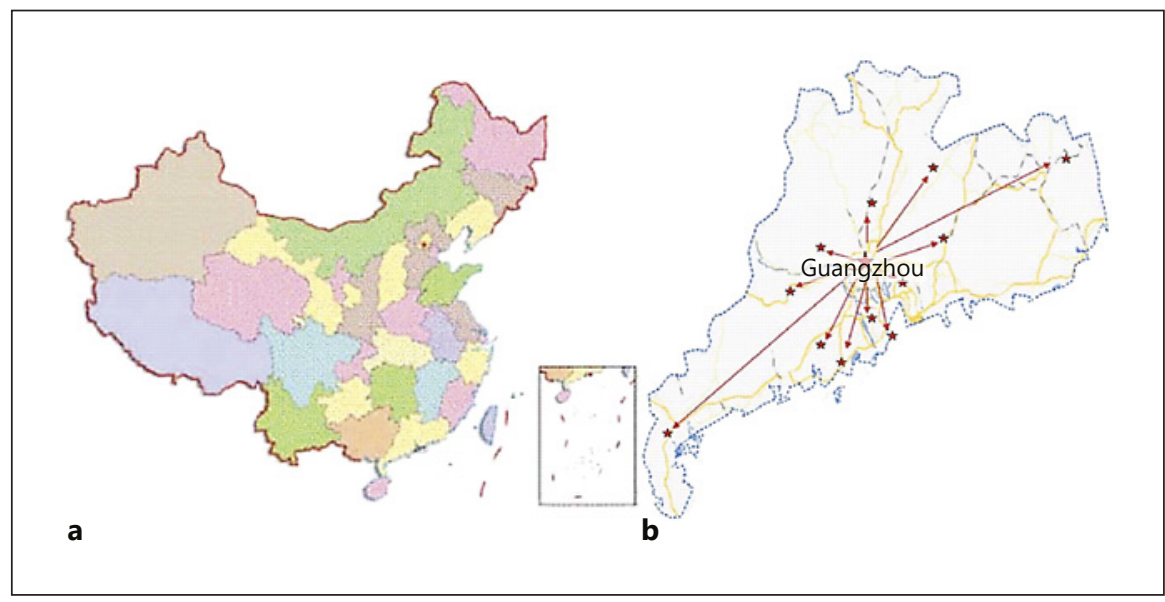

Fig. 1. Map of China (a) and Guangdong Province (b). Guangzhou is the capital city of Guangdong province. Stars indicate the locations of the PD satellite centers.

enhance the application and quality of $\mathrm{PD}$, reducing the episodes of peritonitis as well as decreasing the drop-out rate by providing standard training for the doctors and nurses in satellite hospitals (Fig. 1). Through the satellite PD center program, we provide the PD training courses covering all aspects of PD therapy for nephrologists and nurses who are working in the satellite PD centers. Our satellite program was initiated in January 2008, by the end of 2010, 13 PD units in Guangdong Province had been accepted as satellite centers and 26 specialists as well as 32 nurses participated in the centralized PD technical training organized by our center. Clinical outcome parameters have been significantly improved in the satellite centers after the initiation of the satellite center program. Patients undergoing follow-up satellite PD centers increased from 1,010 in 2007 to 2,544 in 2010, while the annual drop-out rate declined from 28.2 to $18.2 \%$, and the average peritonitis rate declined from 1 episode in 39.4 patient-months to 1 episode in 54.5 patientmonths. The 1-year technical survival rate increased from 88.7 to $93.0 \%$ and the 1 -year patient survival rate climbed from 82.0 to $84.2 \%$. The mean time on PD extended from 16.3 months to 26.1 months (Table 1) [27]. The establishment and enforcement of the PD satellite center project reinforced the management of more than $60 \%$ remote patients at our PD centers and accordingly improved the clinical outcomes. In our PD program, the patient survival rates at 1,2,3, and 5 years for the patients are $94,87,81$, and $64 \%$, respectively. The technical survival rates are $98,95,91$, and $86 \%$, respectively. The incidence of peritonitis is $1 / 68.5$ patientmonths [3]. The model also significantly increased the overall PD treatment level in the satellite hospitals and promotes high-quality PD therapy across the province. According to the latest data, there are more than 10,000 registered PD pa- 
Table 1. Outcome indices of PD patients at all satellite centers, 2007-2009 [27]

\begin{tabular}{lllll}
\hline Variable & \multicolumn{2}{l}{ December } & \multicolumn{2}{c}{$\begin{array}{l}p \text { value } \\
\text { (2009 vs. } \\
\text { 2007) }\end{array}$} \\
\cline { 2 - 4 } & 2007 & 2008 & 2009 & \\
\hline Annual dropout by cause, \% & & & & 0.01 \\
$\quad$ Overall & $28.2 \pm 2.8$ & $23.3 \pm 3.6$ & $17.6 \pm 1.7$ & \\
$\quad$ Death & 51.3 & 48.2 & 39.2 & \\
$\quad$ Transplantation & 26.2 & 22.7 & 18.2 & \\
$\quad$ Transfer to HD & 12.5 & 14.8 & 16.9 & \\
$\quad$ Other & 10.0 & 14.3 & 25.7 & \\
$\quad$ Peritonitis rate (pt-mos./ep.) & $39.4 \pm 2.7$ & $41.3 \pm 4.2$ & $46.2 \pm 3.9$ & \\
1-Year survival, \% & & & & 0.02 \\
$\quad$ Technique & $88.7 \pm 1.5$ & $89.1 \pm 1.9$ & $93.0 \pm 0.8$ & 0.00 \\
$\quad$ Patient & $82.0 \pm 1.1$ & $81.3 \pm 1.6$ & $84.2 \pm 0.9$ & \\
\hline
\end{tabular}

PD, peritoneal dialysis; HD, hemodialysis; pt-mos./ep., patient-months/episode.

tients in Guangdong province, which is the biggest PD population among provinces across China at present. To enhance awareness and clinical level of PD treatment across the country, especially in the rural and remote areas in China, we initiated a National Dialysis Unit Training Program for county hospitals. It is believed that the improved expertise of PD nationwide through the satellite center model and county hospital training model in China will definitely help the utilization and quality of PD, particularly in countryside and remote areas. Our PD satellite center model, named as "Guangzhou model," is highly recognized by peer professionals across the world. Professionals assert that "the establishment and successful implementation of PD satellite centers provide developing and developed countries with an example of the promotion of PD and the improvement of PD quality" [28]. The ISPD position statement on reducing the risk of PD-related infections suggested that "particular attention should be paid to successful programs that deal with patients of low socio-economic status and limited education. One example is a report from southern China, where satellite programs supplying care to rural patients were established using the expertise of a knowledgeable center located at Sun Yat-sen University [27]. That report demonstrates how a structured approach using centers of excellence as models can result in excellent outcomes in outreach programs" [29].

Comprehensive Follow-Up Program for the Management of Remote PD Patients The management of remote PD patients and getting desirable clinical outcomes remain a great challenge. Based on our experience on PD satellite program and advances in telecommunications technologies, we have established a compre- 


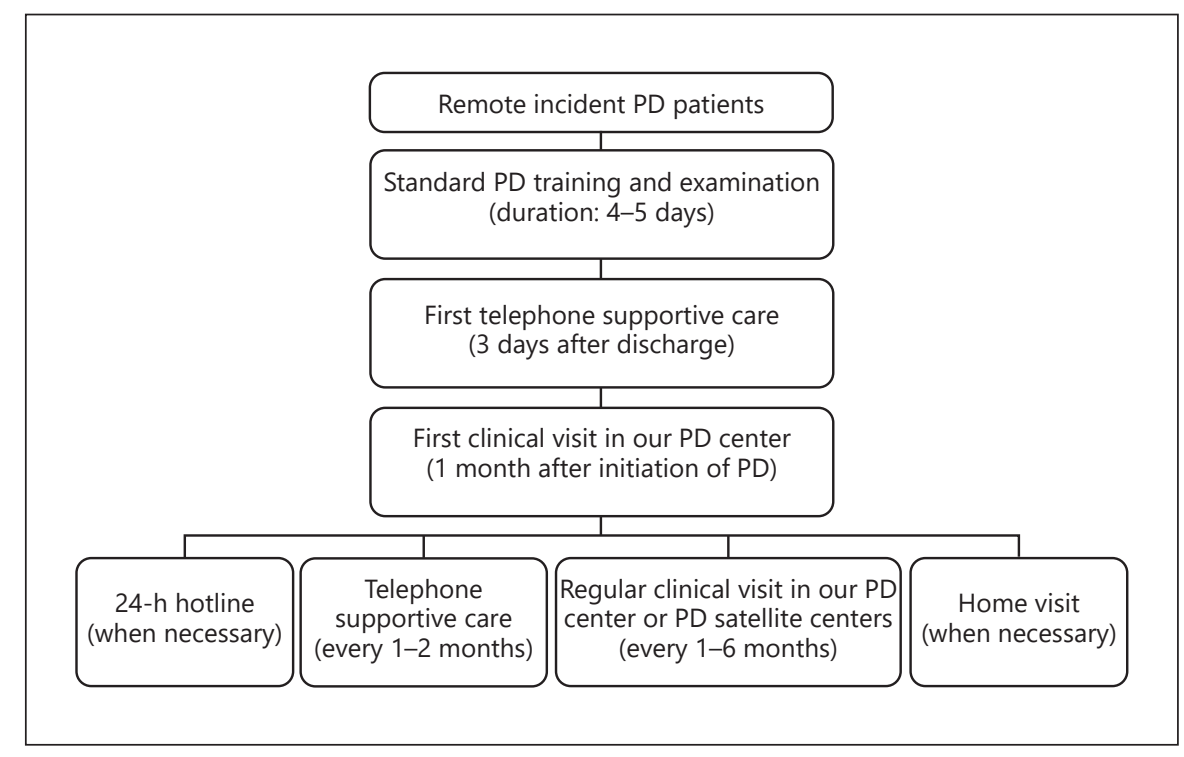

Fig. 2. Comprehensive follow-up program. PD, peritoneal dialysis.

hensive follow-up program to manage our remote PD patients, which include regular PD center visiting care, telephone supportive care, 24-h hotline, and home visits. The PD patients visited our PD center or the nearest PD satellite centers every 1-3 months. The telephonic supportive care was implemented by which the primary PD nurses in our PD center contacted the patients using telephone to assess each $\mathrm{PD}$ patient's condition and to give medical advice every 1-2 months. A 24-h hotline service was provided in case of emergency. Home visit was available to those PD patients who had frequent episodes of peritonitis (Fig. 2). By a median of 35.7 months longitudinal cohort study of 1,005 patients, Kaplan-Meier estimates of patient survival for the remote PD patients are 95.4, 84.7 , and $71.8 \%$ and death-censored technique survival rates were 98.6, 92.3, and $83.4 \%$ at 1,3 , and 5 years, respectively. Peritonitis rate was 0.16 episodes per patient-year (Table 2). Our study indicated that the remote PD patients managed by a comprehensive follow-up program had favorable patient survival, technique survival, and peritonitis rate [30]. In recent years, remote monitoring systems such as mobile phone, telemedicine, and network have been used in the management of remote PD patients $[21,26]$. By our experience, patients living in remote areas might benefit from the remote monitoring services such as the regular telephone supportive care and 24-h hotline services; however, these remote monitoring services could not replace the face-to-face communication between nephrologists and patients completely. We previously demonstrated that a PDC interval of 2 months or less was associated with better clinical outcomes 
Table 2. Outcome indices of remote PD patients [30]

\begin{tabular}{llll}
\hline & 1 year & 3 years & 5 years \\
\hline Patient survival rate, \% & 95.4 & 84.7 & 71.8 \\
Technique survival rate, death censored, \% & 98.6 & 92.3 & 83.4 \\
Peritonitis-free survival rate, \% & 86.4 & 70.8 & 57.6 \\
\hline
\end{tabular}

Analysis by Kaplan-Meier method.

$\mathrm{PD}$, peritoneal dialysis.

in CAPD patients [25]. Increased PDC frequency might modify high morbidity and mortality through rapid identification and treatment of acute conditions and improved management of PD. Therefore, regular time interval of visiting the attending nephrologists in PD centers or satellite PD centers should be encouraged for these patients. The comprehensive follow-up program, which combines the regular time interval of visiting the attending nephrologists with telephonic supportive care, could provide an intensive and all-around medical service and comparable survival for PD patients in non-metropolitan area, which may be a useful way for monitoring $\mathrm{PD}$ patients in remote rural area and improving the quality of $\mathrm{PD}$ as well as clinical outcomes.

In summary, the increase in ESRD population worldwide is of great concern for many countries and has become a worldwide public health problem and puts a heavy medical burden on healthcare resources. Patients living in remote and rural locations could use PD as a home-based and economical therapy option. However, morbidity and mortality in PD patients at remote centers have been reportedly increasing. The establishment and practice of PD satellite center model in China for the management of remote PD patients are a concern for peer professionals across the world. With the support of remote PD center programs, such as the PD satellite center model and telehealth, and so on, home PD therapy have enhanced the PD technology and patient management exercised by quality PD centers, which accordingly benefit more ESRD patients who live in remote areas. Such PD programs can mitigate patients' financial, travel and healthcare inequities and to a certain extent improve the outcomes of the PD patients. Further research is needed to explore more interventions in the management of remote $\mathrm{PD}$ patients.

\section{Disclosure Statement}

The authors declare that they have no conflict of interest. 


\section{Acknowledgements}

The authors thank all nephrologists and nurses in Sun Yat-sen PD center for their excellent management of PD patients.

This work was supported by The National Key Research and Development Program of China (Grant No. 2016YFC0906100), the Natural Science Foundation of China (Grant No. 81570614), Guangdong Science Foundation of China (Grant No. 2014A030313139, 2017A050503003, 2017B020227006), and the Guangzhou Committee of Science and Technology, China (Grant No. 2014Y2-00543, 201704020167).

\section{References}

1 United States Renal Data System. 2018 USRDS Annual Data Report: Incidence, Prevalence, Patient Characteristics, and Treatment Modalities. US Renal Data System. Bethesda, National Institutes of Health, National Institute of Diabetes and Digestive and Kidney Diseases, 2018.

2 Zhang L, Wang F, Wang L, Wang W, Liu B, Liu J, et al: Prevalence of chronic kidney disease in China: a cross-sectional survey. Lancet 2012;379: 815-822.

3 Yu X, Yang X: Peritoneal dialysis in China: meeting the challenge of chronic kidney failure. Am J Kidney Dis 2015;65:147-151.

4 Kramer A, Pippias M, Noordzij M, et al: The European Renal Association - European Dialysis and Transplant Association (ERA-EDTA) registry annual report 2015: a summary. Clin Kidney J 2018;11:108-122.

5 The twelfth biennial health report of the Australian Institute of Health and Welfare 2010: Australian Institute of Health and Welfare URL: http:// www.aihw.gov.au/WorkArea/DownloadAsset. aspx?id=6442452962 [Web Cite Cache ID 6XHRqe4Qg] (accessed March 25, 2015).

6 Chinese Renal Data System (CNRDS). www. CNRDS.NET (accessed October 15, 2018).

7 Eggers PW: Has the incidence of end-stage renal disease in the USA and other countries stabilized? Curr Opin Nephrol Hypertens 2011;20:241-245.

8 Tsai MH, Hsu CY, Lin MY, et al: Incidence, prevalence, and duration of chronic kidney disease in Taiwan: results from a community-based screening program of 106,094 individuals. Nephron 2018;140:175-184.

9 Komenda P, Lavallee B, Ferguson TW, et al: The prevalence of $\mathrm{CKD}$ in rural Canadian indigenous peoples: results from the First Nations Community Based Screening to Improve Kidney Health and Prevent Dialysis (FINISHED) screen, triage, and treat Program. Am J Kidney Dis 2016;68:582-590.
10 Komenda P, Yu N, Leung S, et al: Secular trends in end-stage renal disease requiring dialysis in Manitoba, Canada: a population-based study. CMAJ Open 2015;3:E8-E14.

11 Fan Z, Lackland DT, Lipsitz SR, et al: Geographical patterns of end-stage renal disease incidence and risk factors in rural and urban areas of South Carolina. Health Place 2007;13:179-187.

12 Sanabria M, Devia M, Hernández G, et al: Outcomes of a peritoneal dialysis program in remote communities within Colombia. Perit Dial Int 2015;35:52-61.

13 Chen W, Chen W, Wang H, et al: Prevalence and risk factors associated with chronic kidney disease in an adult population from southern China. Nephrol Dial Transplant 2009;24:1205-1212.

14 Liu Q, Li Z, Wang H, et al: High prevalence and associated risk factors for impaired renal function and urinary abnormalities in a rural adult population from southern China. PLoS One 2012; 7:e47100

15 Smith KB, Humphreys JS, Wilson MG: Addressing the health disadvantage of rural populations: how does epidemiological evidence inform rural health policies and research? Aust J Rural Health 2008;16:56-66.

16 Rucker D, Hemmelgarn BR, Lin M, et al: Quality of care and mortality are worse in chronic kidney disease patients living in remote areas. Kidney Int 2011;79:210-217.

17 Tonelli M, Manns B, Culleton B, et al: Association between proximity to the attending nephrologist and mortality among patients receiving hemodialysis. CMAJ 2007;177:1039-1044.

18 Tonelli M, Hemmelgarn B, Culleton B, et al: Mortality of Canadians treated by peritoneal dialysis in remote locations. Kidney Int 2007;72:10231028.

19 Zhang W, Gong Z, Peng X, et al: Clinical characteristics and outcomes of rural patients with ESRD in Guangxi, China: one dialysis center experience. Int Urol Nephrol 2010;42:195-204. 
20 Thompson S, Gill J, Wang X, Padwal R, Pelletier $\mathrm{R}$, Bello A, et al: Higher mortality among remote compared to rural or urban dwelling hemodialysis patients in the United States. Kidney Int 2012; 82:352-359.

21 Milan Manani S, Crepaldi C, Giuliani A, et al: Remote monitoring of automated peritoneal dialysis improves personalization of dialytic prescription and patient's independence. Blood Purif 2018;46:111-117.

22 Jain AK, Blake P, Cordy P, et al: Global trends in rates of peritoneal dialysis. J Am Soc Nephrol 2012;23:533-544.

23 Lim WH, Boudville N, McDonald SP, et al: Remote indigenous peritoneal dialysis patients have higher risk of peritonitis, technique failure, allcause and peritonitis-related mortality. Nephrol Dial Transplant 2011;26:3366-3372.

24 Mehrotra R, Story K, Guest S, et al: Neighborhood location, rurality, geography, and outcomes of peritoneal dialysis patients in the United States. Perit Dial Int 2012;32:322-323.
25 Yi C, Guo Q, Lin J, et al: Patient-doctor contact interval and clinical outcomes in continuous ambulatory peritoneal dialysis patients. Am J Nephrol 2017;45:346-352.

26 Lunney M, Lee R, Tang K, et al: Impact of telehealth interventions on processes and quality of care for patients with ESRD. Am J Kidney Dis 2018;72:592-600.

27 Jiang Z, Yu X: Advancing the use and quality of peritoneal dialysis by developing a peritoneal dialysis satellite center program. Perit Dial Int 2011;31:121-126.

28 Lo WK: Improving peritoneal dialysis outcomes through networking based on experienced centers of excellence. Perit Dial Int 2011;31:131-133.

29 Piraino B, Bernardini J, Brown E, et al: ISPD position statement on reducing the risks of peritoneal dialysis-related infections. Perit Dial Int 2011;31: 614-630.

30 Yi C, Guo Q, Lin J, et al: Clinical outcomes of remote peritoneal dialysis patients: a retrospective cohort study from a single center in China. Blood Purif 2016;41:100-107.

Xueqing Yu, MD, PhD

Guangdong Provincial People's Hospital

Guangdong Academy of Medical Science, 106th Zhongshan Road II

Guangzhou 510080 (China)

E-Mail yuxq@mail.sysu.edu.cn 